\title{
A Study on the Cost Analysis for the Container Terminal Services based on $\mathrm{ABC}$ Approach
}

\author{
*Dong-Ha Ryu $+{ }^{\dagger}$ Ki-Myung Ahn $\cdot * * Y e o-$ Sang Yoon \\ *Marketing Team Leader, Hutchison Korea Terminals, Busan, Korea \\ + Division of Shipping Management, Professor of Korea Maritime University \\ **General Manager, Korea Maritime News, Busan, Korea
}

\begin{abstract}
Terminal market has rapidly crashed and market rates have taken a sharp plunge. The substantial throughput decrease resulted from the world economic downturn has been a finishing blow to the terminal operators in Busan. Every terminal operator is taking cost saving as its first priority and accelerating structural reform and downsizing. Under the desperate situation, the need of effective cost analysis would be highly required to effectively control operation cost and to develop new services to satisfy the different needs of the customers. Furthermore, terminal operators could reduce unnecessary activities and concentrate their resource on the more cost-effective process through the operation cost analysis. In order to suggest a new framework of the cost control of container terminals, this paper seeks to analyze terminal costs based on $A B C$ approach by processing actual data.
\end{abstract}

Key words : Cost Analysis, Container Terminal Services, Activity Based Costing, Terminal cost, Cost driving factors

\section{Introduction}

This study tried to recognize the importance of cost analysis on the terminal services and suggested $\mathrm{ABC}$ approach to analyze the cost. This kind of cost analysis could offer a fresh perspective on the container terminal services and activities. Terminal operators could understand what is cost effective activity and what activity could be reduced or eliminated through the cost analysis. This analysis and approach could help terminal operators to pursue productivity improvement and enhance operation efficiency. In addition, this approach can be used to develop new services and differentiate the current standardized terminal services. The most important thing is to know a terminal operator's own position and situation. In other words, where it is, where it to go, what it has to do and what it has to solve under the new challenges. Cost analysis of the terminal services could be a good solution for the questions.

\section{Container Market Situation}

Busan port is in keen competition not only with domestic ports such as Incheon, Gwangyang, Ulsan, Pohang and Pyungtaek but also neighboring international ports of China or Japan to become a hub port in the North Asia.

Under the fierce competition, the development of Busan New Port led by the government has resulted in over-supply situation in Busan market and local terminal operators in Busan are struggling for survival. They are placing their top priority on cost saving and downsizing over maintaining service quality or pursing high productivity.

Under these circumstances, it would be highly recommended for terminal operators to systemically analyze their production cost in order to differentiate terminal services, develop new services and effectively control their cost. According to Lee (2009) study, cutthroat competition among the domestic ports will become fiercer considering the downward trend of throughput growth and intensifying competition.

\section{Cost analysis on terminal services based on $A B C$ approach}

\subsection{ABC approach}

$\mathrm{ABC}$ approach has been introduced to improve the traditional costing system in the manufacturing area and it has been extended to the service industries since 1990s. (Han, 2006). Rotch(1990) carried out research to apply

\footnotetext{
* Main Author, btrdh@hktl.com 051)630-8244

+ Corresponding Author, kmahn@hhu.ac.kr 051)410-4385

** clifftop@naver.com 051)442-4369
} 
$\mathrm{ABC}$ approach to the service industries such as Hospital, Railroad, Cruise and Information providing companies by comparing the result of $\mathrm{ABC}$ approach with the traditional costing system.

\subsection{Literature review}

Dater(1991) has proved the usefulness of the ABC approach through the field research in the auto-parts manufacturing industry. Kwon(2005) tried to apply new costing system to analyze the cost structure of railway freight industry in Korea. Hur(2009) carried out a research and analysis in shipping area to study its contribution to the regional economy based on revenue per TEU.

\subsubsection{Cost analysis process}

This study seeks to suggest a useful and practicable approach for cost analysis and performed cost analysis based on the operation data and detailed cost items of the 'H terminal' in Busan based on the five months' sample data. Terminal cost analysis will be done through the following seven processes as shown in the Table 1 .

Table 1 The process of cost analysis

\begin{tabular}{|c|l|}
\hline step & \multicolumn{1}{|c|}{ action } \\
\hline 1 & Defining service products \\
\hline 2 & Defining key activities \\
\hline 3 & $\begin{array}{l}\text { Analyzing the ratio of key activities put into the } \\
\text { defined products }\end{array}$ \\
\hline 4 & Defining cost items \\
\hline 5 & Allocating costs into the key activities \\
\hline 6 & Calculating the cost by products \\
\hline 7 & Verifying the process and allocated cost \\
\hline
\end{tabular}

\subsubsection{Defining service products}

There could be various approaches to recognize terminal services as a product. This paper assorts terminal services based on equipment activity and appointed five representative services as shown in the Table 2 .

There are two kinds of service modes in Busan. Off-dock mode had been a general terminal service in Busan port until mid of 2000 but these days, On-dock service is a prevailing mode and most of shipping lines are using terminals under on-dock service mode. On-dock service could be understood as an special services and terminals provides their customers with more advanced services under on-dock mode.
Table 2 Terminal service products

\begin{tabular}{|c|c|c|c|c|c|c|}
\hline \multirow{2}{*}{$\begin{array}{c}\text { Service } \\
\text { Products }\end{array}$} & \multicolumn{2}{|c|}{ Full } & \multicolumn{2}{c|}{ Empty } & \multicolumn{2}{c|}{ Total } \\
\cline { 2 - 7 } & $20^{\prime}$ & $40^{\prime}$ & $20^{\prime}$ & $40^{\prime}$ & VAN & TEU \\
\hline Local & 116,497 & 92,142 & 32,100 & 32,569 & 273,309 & 398,020 \\
\hline Internal TS & 50,874 & 50,641 & 4,365 & 10,295 & 116,175 & 177,110 \\
\hline Inter terminal TS & 45,042 & 48,788 & 1,318 & 2,372 & 97,521 & 148,682 \\
\hline One time shifting & 52 & 104 & 53 & 96 & 306 & 506 \\
\hline Two times shifting & 2,778 & 6,203 & 1,039 & 2,668 & 12,689 & 21,561 \\
\hline Total & 215,244 & 197,879 & 38,876 & 48,000 & 500,000 & 745,879 \\
\hline
\end{tabular}

* H terminal's actual data

This paper analyzes each operation cost for Off-dock and On-dock mode respectively.

\subsubsection{Defining key activities}

Key activities can be understood as a raw material in the manufacturing industry. Defining key activities is the most important point of this study because the approach and the result of the study could vary with what factors we consider as a raw material to produce invisible products in the terminal industry. This paper recognizes the activity of major equipment as a cost factor and allocates total cost to the key activities. Q/C(Quay Crane), RTGC(Yard Crane) and Y/T(Yard Truck) are major equipment in the terminal and this paper actually counted all their activities used to produce service products forementioned.

\subsection{Analyzing the ratio of key activities put into the defined products}

\subsubsection{Q/C activities}

$\mathrm{Q} / \mathrm{C}$ is used to handle containers or hatch covers during the loading or unloading of local or transshipment(T/S) cargos. (see Table 3)

Table $3 \mathrm{Q} / \mathrm{C}$ activities

\begin{tabular}{|c|c|c|c|c|c|}
\hline \multirow{2}{*}{ Local/TS } & \multicolumn{2}{|c|}{ Shifting } & \multirow{2}{*}{$\begin{array}{c}\text { Hatch } \\
\text { Cover }\end{array}$} & \multirow{2}{*}{ Total } \\
\cline { 3 - 4 } & & 1 times & 2 times & \\
\hline Total & 487,005 & 295 & 12,689 & 8,989 & 508,978 \\
\hline Off-dock & 398,608 & 270 & 12,097 & 6,559 & 417,535 \\
\hline On-dock & 88,397 & 25 & 593 & 2,429 & 91,443 \\
\hline
\end{tabular}

\subsubsection{RTGC activities}

RTGC is used to support Q/C for loading or discharging containers and provide lift on or off services for road transportation vehicles. RTGC also handles 2 time shifting containers (cell-dock-cell restore) or yard marshalling, yard rehandling and lift on/off service for on-dock empty containers. Table 4 includes the activities of similar type of 
equipment such as yard forklift or reach-stacker

Table 4 RTGC activities

\begin{tabular}{|c|c|c|c|c|c|c|c|}
\hline & \multicolumn{2}{|c|}{ Local and TS } & \multirow[b]{2}{*}{\begin{tabular}{c|} 
On- \\
dock \\
lift \\
on/off
\end{tabular}} & \multirow[b]{2}{*}{$\begin{array}{l}2 \text { time } \\
\text { shifting }\end{array}$} & \multicolumn{2}{|c|}{ Yard rehandling } & \multirow[b]{2}{*}{ Total } \\
\hline & \begin{tabular}{|c|}
$\mathrm{Q} / \mathrm{C}$ \\
loading \\
and \\
unloading
\end{tabular} & \begin{tabular}{|c} 
Lift \\
on/off \\
for road \\
vehicles
\end{tabular} & & & $\begin{array}{c}\mathrm{Y} / \mathrm{T} \\
\text { move } \\
(2 \mathrm{TIME})\end{array}$ & \begin{tabular}{|c} 
same \\
block \\
move \\
(1TIME)
\end{tabular} & \\
\hline Total & 487,005 & 370,832 & 13,564 & 1,927 & 27,9 & 242,638 & $1,143,96$ \\
\hline Off-dock & 398,608 & \begin{tabular}{|l}
287,677 \\
\end{tabular} & & 1,580 & 22,913 & 228,771 & \begin{tabular}{|l}
939,549 \\
\end{tabular} \\
\hline On-dock & 88,397 & 83,155 & 13,564 & 347 & 5,081 & 13,867 & 204,411 \\
\hline
\end{tabular}

\subsubsection{Y/T activities}

$\mathrm{Y} / \mathrm{T}$ is used to move containers within terminal area. Table 5 shows the activities of $\mathrm{Y} / \mathrm{T}$ in each case.

Table $5 \mathrm{Y} / \mathrm{T}$ activities

\begin{tabular}{|c|c|c|c|c|c|c|c|}
\hline & \multicolumn{2}{|c|}{ Local and TS } & \multirow[b]{2}{*}{$\begin{array}{l}\text { On- } \\
\text { dock } \\
\text { lift } \\
\text { on/off }\end{array}$} & \multirow[b]{2}{*}{$\begin{array}{l}2 \text { time } \\
\text { shifting }\end{array}$} & \multicolumn{2}{|c|}{ Yard rehandling } & \multirow[b]{2}{*}{ Total } \\
\hline & \begin{tabular}{|c}
$\mathrm{Q} / \mathrm{C}$ \\
loading \\
and \\
unloading \\
\end{tabular} & $\begin{array}{c}\text { Lift } \\
\text { on/off } \\
\text { for road } \\
\text { vehicles }\end{array}$ & & & $\begin{array}{c}\mathrm{Y} / \mathrm{T} \\
\text { move } \\
\text { (2TIME) }\end{array}$ & $\begin{array}{c}\text { same } \\
\text { block } \\
\text { move } \\
\text { (1TIME) }\end{array}$ & \\
\hline Total & 487,005 & & & 12,689 & 13,997 & & 513,69 \\
\hline Off-dock & 398,608 & & & 12,097 & 11,456 & & 422,16 \\
\hline On-dock & 88,397 & & & 593 & 2,541 & & 91,530 \\
\hline
\end{tabular}

\subsubsection{Total activities by products}

Table 6 totaled all activities by products. For one example, Q/C is used 370,832 times to produce Local and Inter terminal TS for five months. 370,832 times of $\mathrm{Q} / \mathrm{C}$ activities can be divided 287,677 times for Off-dock and 83,155 for On-dock. In the meantime, Table 6 shows that $\mathrm{Q} / \mathrm{C}$ is necessarily used to produce all kinds of product. So, $\mathrm{Q} / \mathrm{C}$ activities could be a basic criterion to simplify the Table 6.

Table 6 Total activities by products

\begin{tabular}{|c|c|c|c|c|c|c|c|c|c|}
\hline & \multicolumn{3}{|c|}{ Total } & \multicolumn{3}{c|}{ Off-dock } & \multicolumn{3}{c|}{ On-dock } \\
\cline { 2 - 11 } & Q/C & RTGC & Y/T & Q/C & RTGC & Y/T & Q/C & RTGC & Y/T \\
\hline $\begin{array}{c}\text { Local and } \\
\text { Inter } \\
\text { terminal TS }\end{array}$ & 370,832 & 989,325 & 382,940 & 287,677 & 793,061 & 297,587 & 83,155 & 196,264 & 85,353 \\
\hline $\begin{array}{c}\text { Interna } \\
\text { TS }\end{array}$ & 116,172 & 152,708 & 118,062 & 110,931 & 144,908 & 112,478 & 5,242 & 7,800 & 5,584 \\
\hline $\begin{array}{c}\text { 1 Time } \\
\text { Shifting }\end{array}$ & 295 & & & 270 & & & 25 & & \\
\hline $\begin{array}{c}2 \text { Times } \\
\text { Shifting }\end{array}$ & 12,689 & 1,927 & 12,689 & 12,097 & 1,580 & 12,097 & 593 & 347 & 593 \\
\hline H/cover & 8,989 & & & 6,559 & & & 2,429 & & \\
\hline Total & 508,978 & $1,143,960$ & 513,691 & 417,535 & 939,549 & 422,161 & 91,443 & 204,411 & 91,530 \\
\hline
\end{tabular}

Table 7 shows the ratio of key activities based on one unit of $\mathrm{Q} / \mathrm{C}$ activities by each product. For one example, 1 move of $\mathrm{Q} / \mathrm{C}, 2.76$ move of RTGC and 1.03 move of $\mathrm{Y} / \mathrm{T}$ activities are used to produce one unit of Off-dock Local product.(1 teu of Local cargo)

Table 7 The ratio of key activities per Q/C 1 move

(Unit: \%)

\begin{tabular}{|c|c|c|c|c|c|c|c|c|c|}
\hline & \multicolumn{3}{|c|}{ Total(Average) } & \multicolumn{3}{c|}{ Off-dock } & \multicolumn{3}{c|}{ On-dock } \\
\cline { 2 - 11 } & Q/C & RTGC & Y/T & Q/C & RTGC & Y/T & Q/C & RTGC & Y/T \\
\hline $\begin{array}{c}\text { Local and Inter } \\
\text { terminal TS }\end{array}$ & 1.00 & 2.67 & 1.03 & 1.00 & 2.76 & 1.03 & 1.00 & 2.36 & 1.03 \\
\hline Internal TS & 1.00 & 1.31 & 1.02 & 1.00 & 1.31 & 1.01 & 1.00 & 1.49 & 1.07 \\
\hline 1 Time Shifting & 1.00 & & & 1.00 & & & 1.00 & & \\
\hline 2 Times Shifting & 1.00 & 0.15 & 1.00 & 1.00 & 0.13 & 1.00 & 1.00 & 0.59 & 1.00 \\
\hline H/cover & 1.00 & & & 1.00 & & & 1.00 & & \\
\hline Total(Average) & 1.00 & 2.25 & 1.01 & 1.00 & 2.25 & 1.01 & 1.00 & 2.24 & 1.00 \\
\hline
\end{tabular}

\subsection{Defining Cost Items}

This chapter reviews the terminal cost and classifies the cost items to allocate the cost to the key activities by using five months of actual cost.

As the Table 8 shows the structure of container terminal cost, terminal business is a labor-intensive and capitalintensive industry.

This paper groups the cost items into six categories such as Labor cost (33\%), Terminal rental cost (29.8\%), Nonoperating expense (Depreciation, Amortization \& Interest expense, 21.1\%), Power and Fuel (3.8\%), Equipment maintenance (3.5\%) and Other overhead costs(8.8\%).

In the meantime, this paper divides each cost group into direct costs and overhead. Overhead costs are apportioned to the key activities based on the ratio of direct costs.

Table 8 Actual terminal cost

\begin{tabular}{|c|c|c|}
\hline Item & M/Krw & Ratio \\
\hline Wage \& salary (including pension cost) & 11,666 & $28.5 \%$ \\
\hline Fringe benefit & 1,851 & $4.5 \%$ \\
\hline Equipment maintenance & 1,431 & $3.5 \%$ \\
\hline Power \& Fuel & 1,566 & $3.8 \%$ \\
\hline Depreciation & 1,892 & $4.6 \%$ \\
\hline Terminal Rental Cost & 12,216 & $29.8 \%$ \\
\hline Amortization \& Interest expense & 6,743 & $16.5 \%$ \\
\hline Communication cost & 32 & $0.1 \%$ \\
\hline Consumable cost & 76 & $0.2 \%$ \\
\hline Transportation & 661 & $1.6 \%$ \\
\hline IT cost & 210 & $0.5 \%$ \\
\hline Others & 2,349 & $5.7 \%$ \\
\hline TAX & 291 & $0.7 \%$ \\
\hline Total Cost & 40,984 & $100.0 \%$ \\
\hline
\end{tabular}

* H terminal's actual cost for five months. 


\subsubsection{Labor Cost}

Labor cost is about 33\% of total terminal cost. Q/C, RTGC and Y/T drivers' wage is allocated to the key activities as a direct labor cost. Other labor costs such as office workers' and other field workers' wage and fringe benefit are apportioned as a overhead cost.

Table 9 Labor cost (Unit: Thousand/Krw)

\begin{tabular}{|c|c|c|c|c|}
\hline & Items & Staffs & Avg. wage & Total wage \\
\hline \multirow{4}{*}{$\begin{array}{c}\text { Direct } \\
\text { Labor } \\
\text { Cost }\end{array}$} & Q/C Driver & 53 & 22,703 & $1,203,283$ \\
\cline { 2 - 5 } & RTGC Driver & 119 & 22,703 & $2,701,710$ \\
\cline { 2 - 5 } & Y/T Driver & 135 & 22,703 & $3,064,965$ \\
\cline { 2 - 5 } & S/Total & 307 & & $6,969,957$ \\
\hline \multirow{4}{*}{\begin{tabular}{c} 
Overhead \\
\cline { 2 - 5 }
\end{tabular}} & Other staffs & 249 & 15,577 & $3,878,735$ \\
\cline { 2 - 5 } & Office workers & & & 816,960 \\
\cline { 2 - 5 } & Fringe benefit & & & $1,851,179$ \\
\cline { 2 - 5 } & S/Total & 249 & 26,293 & $6,546,874$ \\
\hline \multicolumn{2}{|c|}{ Total Labor Cost } & 556 & 24,311 & $13,516,831$ \\
\hline
\end{tabular}

\subsubsection{Power and Fuel}

Power and Fuel cost is about 3.8\% of total cost and consists of Power, Fuel and Lubricant oil.

Table 10 Power and Fuel

(Unit: Thousand/Krw)

\begin{tabular}{|c|c|c|c|c|c|c|c|}
\hline & \multicolumn{2}{|c|}{ Direct } & Power and Fuel & \multicolumn{3}{|c|}{ Overhead cost } & \multirow{2}{*}{ Total } \\
\cline { 2 - 7 } & Q/C & RTGC & Y/T & $\begin{array}{c}\text { Reefer } \\
\text { plugging }\end{array}$ & $\begin{array}{c}\text { Light } \\
\text { tower }\end{array}$ & Others & \\
\hline Power & 175,120 & 0 & 0 & 162,492 & 81,709 & & 419,321 \\
\hline Fuel & 0 & 803,580 & 299,191 & 0 & 0 & 0 & $1,102,771$ \\
\hline Lubricant & 9,705 & 20,788 & 8,497 & 0 & 0 & 4,918 & 43,908 \\
\hline Total & 184,825 & 824,368 & 307,688 & 162,492 & 81,709 & 4,918 & $1,566,000$ \\
\hline
\end{tabular}

\subsubsection{Equipment Maintenance}

Equipment maintenance cost is about 3.5\% of total cost and allocated into the key activities based on the same logic with other cost groups.

Table 11 Equipment maintenance (Unit: Thousand/Krw)

\begin{tabular}{|c|c|c|c|c|c|}
\hline & \multicolumn{3}{|c|}{ Direct Cost } & Overhead & \multirow{2}{*}{ Total } \\
\cline { 2 - 5 } & $\mathrm{Q} / \mathrm{C}$ & RTGC & Y/T & Others & \\
\hline Repair & 88,877 & 129,032 & 41,187 & 23,749 & 282,845 \\
\hline Construction & 243,550 & 34,299 & 111,251 & 34,180 & 423,279 \\
\hline Parts & 164,109 & 242,305 & 191,782 & 64,266 & 662,463 \\
\hline $\begin{array}{c}\text { Consumable } \\
\text { items }\end{array}$ & 10,808 & 8,327 & 4,564 & 38,443 & 62,143 \\
\hline Total & 507,344 & 413,964 & 348,784 & 160,638 & $1,430,730$ \\
\hline
\end{tabular}

\subsubsection{Non-operating Expense}

Non-operating expense is about $21.1 \%$ of total cost and allocated into the key activities based on the same logic with other cost groups.

Table 12 Non-operating expense (Unit: Thousand/Krw)

\begin{tabular}{|c|c|c|c|c|c|}
\hline \multirow{2}{*}{ Cost } & \multicolumn{3}{|c|}{ Direct cost } & Overhead & \multirow{2}{*}{ Total } \\
\cline { 2 - 5 } & Q/C & RTGC & YD Y/T & Others & \\
\hline & 980,934 & 338,037 & 185,882 & 387,316 & $1,892,169$ \\
\hline
\end{tabular}

\subsubsection{Terminal Rental Cost}

Terminal rental cost is about $29.8 \%$ of total cost and it consists of rental charge for land and rental charge for buildings and facilities.

Table 13 Terminal Rental Cost

(Unit: Thousand/Krw)

\begin{tabular}{|c|c|c|}
\hline $\begin{array}{c}\text { Charge for } \\
\text { Land }\end{array}$ & $\begin{array}{c}\text { Charge for Buildings } \\
\text { and facilities }\end{array}$ & Total \\
\hline $11,908,649$ & 307,338 & $' 12,215,987$ \\
\hline
\end{tabular}

This paper classifies all the terminal rental cost as a overhead but it is required to divide the cost into the rental charge for land and the others because the land charge should be more allocated into On-dock services mode.

Off-dock containers are, on average, using terminal yard for 3.38 days but On-dock containers are staying at terminal yard for 5.52 days. So, this paper allocates the land charge to Off-dock and On-dock containers based on their average dwell time within terminal area.

Table 14 Average dwell time by service mode

\begin{tabular}{|c|c|c|c|c|c|}
\hline \multicolumn{3}{|c|}{ Total } & \multicolumn{3}{|c|}{ On-dock } \\
\hline $\begin{array}{l}\text { (A) } \\
\text { Total } \\
\text { TEU }\end{array}$ & $\begin{array}{c}\text { (B)Avg. } \\
\text { Dwell } \\
\text { time } \\
\text { (Day5) }\end{array}$ & $(\mathrm{A}) \times(\mathrm{B})$ & $\begin{array}{c}(\mathrm{A}) \\
\text { Total } \\
\text { TEU }\end{array}$ & $\begin{array}{c}\text { (B)Avg } \\
\text { Dwell } \\
\text { time } \\
\text { (Day5) }\end{array}$ & $(\mathrm{A}) \times(\mathrm{B})$ \\
\hline 500,000 & 3.81 & $1,905,000$ & 99,991 & 5.52 & 551,950 \\
\hline \multicolumn{3}{|c|}{ Off-dock } & \multicolumn{3}{|c|}{ Ratio for allocation } \\
\hline \multirow{2}{*}{$\begin{array}{l}\text { (A) } \\
\text { Total } \\
\text { TEU }\end{array}$} & \multirow{2}{*}{$\begin{array}{c}\text { (B)Avg. } \\
\text { Dwell } \\
\text { time } \\
\text { (Day5) }\end{array}$} & \multirow{2}{*}{$\begin{array}{c}(\mathrm{A}) \\
\times \\
(\mathrm{B})\end{array}$} & \multicolumn{2}{|c|}{ Off-dock } & On-dock \\
\hline & & & \multirow{2}{*}{\multicolumn{2}{|c|}{$71.0 \%$}} & \multirow{2}{*}{$29.0 \%$} \\
\hline 400,009 & 3.38 & $1,352,030$ & & & \\
\hline
\end{tabular}




\subsubsection{Other Overhead Costs}

The other costs except above five categories are grouped as a other overhead costs, which is about $8.8 \%$ of total cost and allocated to the key activities based on the ratio of direct cost.

Table 15 Other overhead costs (Unit: Thousand/Krw)

\begin{tabular}{|c|c|c|}
\hline Items & Costs & Ratio of total cost \\
\hline Communication & 32 & $0.1 \%$ \\
\hline Consumable cost & 76 & $0.2 \%$ \\
\hline Transportation & 661 & $1.6 \%$ \\
\hline IT cost & 210 & $0.5 \%$ \\
\hline Others & 2,349 & $5.7 \%$ \\
\hline TAX & 291 & $0.7 \%$ \\
\hline Total & 3,620 & $8.83 \%$ \\
\hline
\end{tabular}

\subsection{Cost Allocation}

After defining the terminal cost item, this step will allocate the costs into the key activities and calculate unit costs of every cost item by the key activities.

\subsubsection{Labor Cost Allocation}

This study allocates overhead labor cost to the key activities based on the ratio of direct labor cost.

Table 16 Direct labor cost

\begin{tabular}{|c|c|c|c|c|}
\hline & $\begin{array}{c}\text { Key } \\
\text { activities }\end{array}$ & $\begin{array}{c}\text { (A)Total labor } \\
\text { cost(000 Krw) }\end{array}$ & $\begin{array}{c}\text { (B)Total units of } \\
\text { key activities }\end{array}$ & $\begin{array}{c}\text { (A/B) Unit } \\
\text { cost(Krw) }\end{array}$ \\
\hline \multirow{4}{*}{$\begin{array}{c}\text { Direct } \\
\text { cost }\end{array}$} & Q/C & $1,203,283$ & 508,978 & 2,364 \\
\cline { 2 - 5 } & RTGC & $2,701,710$ & $1,143,960$ & 2,362 \\
\cline { 2 - 5 } & Y/T & $3,064,965$ & 513,691 & 5,967 \\
\cline { 2 - 5 } & Total & $6,969,957$ & $2,166,629$ & 3,217 \\
\hline
\end{tabular}

As forementioned, direct labor cost is the wage of equipment drivers and overhead is the wage of other staffs such as office workers and other field workers or fringe benefits.

In the meantime, on-dock mode is a special service compared with the off-dock mode. To provide on-dock services with shipping lines, dedicated on-dock resources are required such as on-dock staffs, office, facilities and so forth. Accordingly, this paper divided the total overhead cost into off-dock and on-dock based on the $\mathrm{Q} / \mathrm{C}$ ratio, $82 \%$ for off-dock and 18\% for on-dock, and allocates each overhead to the key activities based on the ratio of direct labor cost.
Table 17 Overhead labor cost by off-dock and on-dock

\begin{tabular}{|c|c|c|c|c|c|}
\hline & $\begin{array}{c}\text { Key } \\
\text { activities }\end{array}$ & $\begin{array}{c}\text { (A) Overhead } \\
\text { cost } \\
(000 \mathrm{Krw})\end{array}$ & $\begin{array}{c}\text { (B)Total } \\
\text { units of key } \\
\text { activities }\end{array}$ & $\begin{array}{l}\text { (C)The } \\
\text { ratio of } \\
\text { direct cost }\end{array}$ & $\begin{array}{c}(\mathrm{A} * \mathrm{C} / \mathrm{B}) \\
\text { Unit cost } \\
(\mathrm{Krw})\end{array}$ \\
\hline \multirow{3}{*}{$\begin{array}{c}\text { Total } \\
\text { overhead }\end{array}$} & $\mathrm{Q} / \mathrm{C}$ & \multirow{3}{*}{$6,546,874$} & 508,978 & $17 \%$ & 2,221 \\
\hline & RTGC & & $1,143,960$ & $39 \%$ & 2,218 \\
\hline & $\mathrm{Y} / \mathrm{T}$ & & 513,691 & $44 \%$ & 5,604 \\
\hline \multirow{3}{*}{ Off-dock } & $\mathrm{Q} / \mathrm{C}$ & \multirow{3}{*}{$5,100,303$} & 417,535 & $17 \%$ & 2,109 \\
\hline & RTGC & & 939,549 & $39 \%$ & 2,104 \\
\hline & $\mathrm{Y} / \mathrm{T}$ & & 422,161 & $44 \%$ & 5,313 \\
\hline \multirow{3}{*}{ On-dock } & $\mathrm{Q} / \mathrm{C}$ & \multirow{3}{*}{$1,446,571$} & 91,443 & $17 \%$ & 2,731 \\
\hline & RTGC & & 204,411 & $39 \%$ & 2,743 \\
\hline & $\mathrm{Y} / \mathrm{T}$ & & 91,530 & $44 \%$ & 6,950 \\
\hline
\end{tabular}

3.6.2. Power and Fuel Allocation

Table 18 Direct power and fuel cost

\begin{tabular}{|c|c|c|c|c|c|c|c|c|}
\hline & \multicolumn{3}{|c|}{$\begin{array}{c}\text { Kroct } \\
\text { Krw) }\end{array}$} & \multicolumn{2}{c|}{$\begin{array}{c}\text { (B)Total } \\
\text { unit of key }\end{array}$} & \multicolumn{3}{c|}{ (A/B)Unit cost (Krw) } \\
\cline { 2 - 10 } & Power & Fuel & $\begin{array}{c}\text { Lubri } \\
\text {-cant }\end{array}$ & $\begin{array}{c}\text { Po } \\
\text { activities }\end{array}$ & $\begin{array}{c}\text { Por } \\
\text { wer }\end{array}$ & Fuel & $\begin{array}{c}\text { Lubri } \\
\text {-cant }\end{array}$ & Total \\
\hline Q/C & 175,120 & & 9,705 & 508,978 & 344 & & 19 & 363 \\
\hline RTGC & & 803,580 & 20,788 & $1,143,960$ & & 702 & 18 & 721 \\
\hline Y/T & & 299,191 & 8,497 & 513,691 & & 582 & 17 & 599 \\
\hline Total & 175,120 & $1,102,771$ & 38,990 & & & & & \\
\hline
\end{tabular}

Table 19 Overhead power and fuel cost

\begin{tabular}{|c|c|c|c|c|c|c|c|}
\hline & \multicolumn{4}{|c|}{ (A)Overhead (000 Krw) } & \multirow{2}{*}{$\begin{array}{l}\text { (B) Total } \\
\text { unit of key } \\
\text { activities }\end{array}$} & \multirow{2}{*}{$\begin{array}{l}\text { (C) Ratio } \\
\text { of direct } \\
\text { cost }\end{array}$} & \multirow{2}{*}{$\begin{array}{c}(\mathrm{A} * \mathrm{C} / \mathrm{B}) \\
\text { Unit cost } \\
(\mathrm{Krw})\end{array}$} \\
\hline & \begin{tabular}{|c|} 
power for \\
reefer
\end{tabular} & $\begin{array}{l}\text { Light } \\
\text { tower }\end{array}$ & etc & Total & & & \\
\hline $\mathrm{Q} / \mathrm{C}$ & \multirow{3}{*}{162,492} & \multirow{3}{*}{81,709} & \multirow{3}{*}{4,918} & \multirow{3}{*}{249,119} & 508,978 & $14.0 \%$ & 69 \\
\hline RTGC & & & & & $1,143,960$ & $62.6 \%$ & 136 \\
\hline $\mathrm{Y} / \mathrm{T}$ & & & & & 513,691 & $23.4 \%$ & 113 \\
\hline
\end{tabular}

\subsubsection{Equipment Maintenance Allocation}

Table 20 Equipment maintenance cost

\begin{tabular}{|c|c|c|c|c|c|c|}
\hline & $\begin{array}{c}\text { (A)Direct } \\
\text { cost } \\
(000 \mathrm{Krw})\end{array}$ & $\begin{array}{l}\text { (B)Total } \\
\text { unit of key } \\
\text { activities }\end{array}$ & $\begin{array}{c}(\mathrm{A} / \mathrm{B}) \\
\text { Unit cost } \\
\text { (Krw) }\end{array}$ & $\begin{array}{c}(\mathrm{C}) \\
\text { Overhead } \\
\text { (000Krw) }\end{array}$ & $\begin{array}{l}\text { (D) Ratio } \\
\text { of direct } \\
\text { cost }\end{array}$ & $\begin{array}{c}(\mathrm{C} * \mathrm{D} / \mathrm{B}) \\
\text { Unit cost } \\
\text { (Krw) }\end{array}$ \\
\hline $\mathrm{Q} / \mathrm{C}$ & 507,344 & 508,978 & 997 & \multirow{3}{*}{160,6358} & $40 \%$ & 126 \\
\hline RTGC & 413,964 & $1,143,960$ & 362 & & $33 \%$ & 46 \\
\hline $\mathrm{Y} / \mathrm{T}$ & 348,784 & 513,691 & 679 & & $27 \%$ & $\cdot 86$ \\
\hline
\end{tabular}

\subsubsection{Non-operating Expense Allocation}

Non-operating expense includes depreciation, Amortization and Interest expense. Depreciation for each equipment is allocated into the key activities as a direct cost. 
Table 21 Depreciation cost

\begin{tabular}{|c|c|c|c|c|c|c|}
\hline & $\begin{array}{c}\text { (A)Direct } \\
\text { cost } \\
(000 \mathrm{Krw}) \\
\end{array}$ & \begin{tabular}{|c|} 
(B)Total \\
unit of key \\
activities
\end{tabular} & \begin{tabular}{|c|}
$(\mathrm{A} / \mathrm{B})$ \\
Unit cost \\
(Krw) \\
\end{tabular} & \begin{tabular}{|c|}
$\mathrm{C})$ \\
Overhead \\
$($ (000Krw) \\
\end{tabular} & \begin{tabular}{|c|}
$\begin{array}{c}\text { (D) Ratio } \\
\text { of direct } \\
\text { cost }\end{array}$ \\
\end{tabular} & \begin{tabular}{|c|}
$\mathrm{C} * \mathrm{D} / \mathrm{B})$ \\
Unit cost \\
$(\mathrm{Krw})$ \\
\end{tabular} \\
\hline $\mathrm{Q} / \mathrm{C}$ & 980,934 & & 1,927 & \multirow{3}{*}{ 387,316 } & $65 \%$ & 496 \\
\hline 2TGC & 338,037 & $1,143,960$ & 95 & & $22 \%$ & 76 \\
\hline $\mathrm{Y} / \mathrm{T}$ & 185,882 & 513,691 & 362 & & $12 \%$ & 93 \\
\hline
\end{tabular}

In the meantime, Amortization and Interest expense are totally classified as an overhead cost and allocated into the key activities based on the ratio of total direct costs excluding labor cost.

Table 22 Amortization and Interest expense

\begin{tabular}{|c|c|c|c|c|c|}
\hline \multirow{2}{*}{$\begin{array}{c}\text { (A)Amortization } \\
\text { and Interest } \\
\text { (000 Krw) }\end{array}$} & $\begin{array}{c}\text { (B)Total } \\
\text { unit of } \\
\text { key } \\
\text { activities }\end{array}$ & $\begin{array}{c}\text { Allocation(000 Krw) } \\
\text { Total direct cost } \\
\text { (Excluding } \\
\text { labor cost) }\end{array}$ & $\begin{array}{c}\text { (C) } \\
\text { Ratio }\end{array}$ & $\begin{array}{c}\text { Unit cost } \\
\text { (Krw) }\end{array}$ \\
\hline Q/C & \multirow{3}{*}{$6,742,943$} & 508,978 & $1,673,104$ & $41 \%$ & 5,417 \\
\cline { 1 - 3 } RTGC & $1,143,960$ & $1,576,368$ & $39 \%$ & 2,271 \\
\cline { 1 - 3 } Y/T & & 513,691 & 842,354 & $21 \%$ & 2,702 \\
\hline
\end{tabular}

\subsubsection{Terminal Rental Cost Allocation}

Terminal rental cost is classified as an overhead cost and allocated into the key activities based on the ratio of total direct costs excluding labor cost. Total rental charge for land (Krw 11,908,649) is divided into off-dock and on-dock based on the ratio of the Table 23. $71 \%$ for off-dock and $29 \%$ for on-dock)

Table 23 Terminal rental charge for land - Off-dock

\begin{tabular}{|c|c|c|c|c|c|}
\hline \multirow{2}{*}{$\begin{array}{c}\text { (A)Charge } \\
\text { for land } \\
\text { (000 Krw) }\end{array}$} & $\begin{array}{c}\text { (B) Unit of } \\
\text { key ctivities } \\
\text { (Off-dock) }\end{array}$ & $\begin{array}{c}\text { Allocation (000 Krw) } \\
\text { Total direct cost } \\
\text { (Excluding } \\
\text { labor cost) }\end{array}$ & $\begin{array}{c}\text { (C) } \\
\text { Ratio }\end{array}$ & $\begin{array}{c}\text { Unit cost } \\
\text { (Krw) }\end{array}$ \\
\hline Q/C & \multirow{2}{*}{$8,456,423$} & 417,535 & $1,673,104$ & $41 \%$ & 8,281 \\
\cline { 1 - 4 } RTGC & 939,549 & $1,576,368$ & $39 \%$ & 3,467 \\
\cline { 4 - 5 } Y/T & 422,161 & 842,354 & $21 \%$ & 4,124 \\
\hline
\end{tabular}

Table 24 Terminal rental charge for land - On-dock

\begin{tabular}{|c|c|c|c|c|c|}
\hline & \multirow{2}{*}{$\begin{array}{l}\text { (A)Charge } \\
\text { for land } \\
\text { (000 Krw) }\end{array}$} & \multirow{2}{*}{$\begin{array}{l}\text { (B) Unit of } \\
\text { key activities } \\
\text { (On-dock) }\end{array}$} & \multicolumn{2}{|c|}{ Allocation (000 Krw) } & \multirow{2}{*}{$\begin{array}{l}(\mathrm{A} * \mathrm{C} / \mathrm{B}) \\
\text { Unit cos } \\
(\mathrm{Krw})\end{array}$} \\
\hline & & & $\begin{array}{c}\text { Total direct cost } \\
\text { (Excluding labor cost) }\end{array}$ & \begin{tabular}{|c|} 
(C) \\
Ratio
\end{tabular} & \\
\hline $\mathrm{Q} / \mathrm{C}$ & \multirow{3}{*}{$3,452,226$} & 91,443 & $1,673,104$ & $41 \%$ & 15,437 \\
\hline$\overline{\mathrm{RTGC}}$ & & 204,411 & $1,576,368$ & $39 \%$ & 6,506 \\
\hline $\mathrm{Y} / \mathrm{T}$ & & 91,530 & 842,354 & $21 \%$ & 7,765 \\
\hline
\end{tabular}

Table 25 Terminal rental charge for buildings and facilities

\begin{tabular}{|c|c|c|c|c|c|}
\hline & \multirow{2}{*}{$\begin{array}{l}\text { (A)Charge for } \\
\text { buildings and } \\
\text { facilities } \\
\text { (000 Krw) }\end{array}$} & \multirow[b]{2}{*}{$\begin{array}{l}\text { (B) Total } \\
\text { unit of key } \\
\text { activities }\end{array}$} & \multicolumn{2}{|c|}{ Allocation (Krw) } & \multirow[b]{2}{*}{$\begin{array}{l}(\mathrm{A} * \mathrm{C} / \mathrm{B}) \\
\text { Unit cost } \\
(\mathrm{Krw})\end{array}$} \\
\hline & & & $\begin{array}{c}\text { Total direct cost } \\
\text { (Excluding labor } \\
\text { cost) }\end{array}$ & $\begin{array}{c}(\mathrm{C}) \\
\text { Ratio }\end{array}$ & \\
\hline $\mathrm{Q} / \mathrm{C}$ & \multirow{3}{*}{307,338} & 508,978 & $1,673,104$ & $41 \%$ & 247 \\
\hline RTGC & & $1,235,403$ & $1,576,368$ & $39 \%$ & 96 \\
\hline $\mathrm{Y} / \mathrm{T}$ & & 718,102 & 842,354 & $21 \%$ & 88 \\
\hline
\end{tabular}

3.6.6 Other Overhead Costs

Table 26 Other overhead costs

\begin{tabular}{|c|c|c|c|c|c|}
\hline & \multirow[b]{2}{*}{$\begin{array}{c}\text { (A)Other } \\
\text { overhead } \\
\text { (Krw) }\end{array}$} & \multirow[b]{2}{*}{$\begin{array}{l}\text { (B) Total } \\
\text { unit of key } \\
\text { activities }\end{array}$} & \multicolumn{2}{|c|}{ Allocation $(000 \mathrm{Krw})$} & \multirow[b]{2}{*}{$\begin{array}{c}(\mathrm{A} * \mathrm{C} / \mathrm{B}) \\
\text { Unit cost } \\
(\text { Krw })\end{array}$} \\
\hline & & & $\begin{array}{c}\text { Total direct cost } \\
\text { (Excluding labor } \\
\text { cost) }\end{array}$ & $\begin{array}{c}(\mathrm{C}) \\
\text { Ratio } \\
\end{array}$ & \\
\hline $\mathrm{Q} / \mathrm{C}$ & \multirow{3}{*}{$3,619,731$} & 508,978 & $1,673,104$ & $41 \%$ & 2,908 \\
\hline RTGC & & $1,143,960$ & $1,576,368$ & $39 \%$ & 1,219 \\
\hline $\mathrm{Y} / \mathrm{T}$ & & 513,691 & 842,354 & $21 \%$ & 1,451 \\
\hline
\end{tabular}

3.6.7 Totaling up the unit cost by the key activity

Table 27 Total unit cost

\begin{tabular}{|c|c|c|c|c|c|c|c|c|c|}
\hline \multirow{2}{*}{$\begin{array}{c}\text { Cost } \\
\text { item }\end{array}$} & \multicolumn{3}{|c|}{ Average } & \multicolumn{3}{c|}{ Off-dock } & \multicolumn{3}{c|}{ On-dock } \\
\cline { 2 - 11 } & Q/C & RTGC & Y/T & Q/C & RTGC & Y/T & Q/C & RTGC & Y/T \\
\hline Labor & 4,585 & 4,580 & 11,571 & 4,473 & 4,466 & 11,279 & 5,095 & 5,105 & 12,916 \\
\hline $\begin{array}{c}\text { Power\& } \\
\text { Fuel }\end{array}$ & 432 & 857 & 712 & 432 & 857 & 712 & 432 & 857 & 712 \\
\hline $\begin{array}{c}\text { Main } \\
\text {-tenance }\end{array}$ & 1,123 & 408 & 765 & 1,123 & 408 & 765 & 1,123 & 408 & 765 \\
\hline $\begin{array}{c}\text { Non- } \\
\text { operating }\end{array}$ & 7,840 & 2,642 & 3,157 & 7,840 & 2,642 & 3,157 & 7,840 & 2,642 & 3,157 \\
\hline Rental & 9,814 & 4,106 & 4,861 & 8,528 & 3,563 & 4,212 & 15,684 & 6,602 & 7,853 \\
\hline Others & 2,908 & 1,219 & 1,451 & 2,908 & 1,219 & 1,451 & 2,908 & 1,219 & 1,451 \\
\hline Total & 26,702 & 13,812 & 22,516 & 25,304 & 13,155 & 21,576 & 33,082 & 16,833 & 26,854 \\
\hline
\end{tabular}

Above table 27 integrates all unit costs and shows the result of cost allocation. For one example, the unit cost of Q/C is Krw 26,702 and it consists of labor cost (Krw 4,585), Power and Fuel (Krw 432), Equipment maintenance (Krw 123), Non-operating cost (Krw 7,840), Terminal rental cost (Krw 9,814) and other overhead (Krw 2,908)

Table 28 simplifies the unit cost based on the Table 27. The unit cost actually means a unit cost per VAN.

Table 28 Unit cost by the key activities(Major Equipment)

\begin{tabular}{|c|c|c|c|c|c|c|}
\hline \multirow{2}{*}{} & \multicolumn{2}{|c|}{ Total(Average) } & \multicolumn{2}{c|}{ Off-dock } & \multicolumn{2}{c|}{ On-dock } \\
\cline { 2 - 7 } & VAN & TEU & VAN & TEU & VAN & TEU \\
\hline Q/C & 26,702 & 17,921 & 25,304 & 16,983 & 33,082 & 22,202 \\
\hline RTGC & 13,812 & 9,270 & 13,155 & 8,829 & 16,833 & 11,297 \\
\hline Y/T & 22,516 & 15,112 & 21,576 & 14,481 & 26,854 & 18,023 \\
\hline Total & 63,030 & 43,302 & 60,035 & 41,292 & 76,769 & 51,523 \\
\hline
\end{tabular}

So, the unit cost per VAN can be converted into the unit cost per TEU by multiplying it by 1.49 (VAN-TEU ratio of 1: 1.49 , namely, 500,000 VAN $=745,879$ TEU in Table 2)

\section{7 Cost Calculation by Service Products}

As the Table 28 indicates, the process of cost analysis has been finalized and all unit costs by equipment are ready 
to calculate the cost of the defined service products in the Table 2. The cost of each service product defined in the Table 2 can be calculated by multiplying the unit cost in the Table 28 with the ratio of key activities by products in the Table 7 as shown the result in the Table 29 .

Table 29 The cost of service products per VAN

(Unit: Krw/VAN)

\begin{tabular}{|c|c|c|c|c|c|c|c|c|c|}
\hline \multirow{2}{*}{ Products } & \multicolumn{3}{|c|}{ Total(Average) } & \multicolumn{3}{c|}{ Off-dock } & \multicolumn{3}{c|}{ On-dock } \\
\cline { 2 - 11 } & $\mathrm{Q} / \mathrm{C}$ & RTGC & $\mathrm{Y} / \mathrm{T}$ & $\mathrm{Q} / \mathrm{C}$ & $\mathrm{RTGC}$ & $\mathrm{Y} / \mathrm{T}$ & $\mathrm{Q} / \mathrm{C}$ & $\mathrm{RTGC}$ & $\mathrm{Y} / \mathrm{T}$ \\
\hline $\begin{array}{c}\text { Local and } \\
\text { Inter } \\
\text { terminal } \\
\text { TS }\end{array}$ & 26,702 & 36,849 & 23,252 & 25,304 & 36,266 & 22,319 & 33,082 & 39,730 & 27,564 \\
\hline $\begin{array}{c}\text { Internal } \\
\text { TS }\end{array}$ & 26,702 & 18,156 & 22,883 & 25,304 & 17,184 & 21,877 & 33,082 & 25,048 & 28,611 \\
\hline $\begin{array}{c}\text { 1 Time } \\
\text { Shifting }\end{array}$ & 26,702 & - & - & 25,304 & - & - & 33,082 & - & - \\
\hline $\begin{array}{c}\text { 2 Times } \\
\text { Shifting }\end{array}$ & 26,702 & 2,097 & 22,516 & 25,304 & 1,718 & 21,576 & 33,082 & 9,851 & 26,854 \\
\hline H/cover & 26,702 & - & - & 25,304 & - & - & 33,082 & - & - \\
\hline Total & 26,702 & 31,044 & 22,725 & 25,304 & 29,602 & 21,815 & 33,082 & 37,628 & 26,879 \\
\hline
\end{tabular}

Meanwhile, it is common for terminal operators to use TEU based criteria as a yardstick. As forementioned, VAN based result can be converted into TEU based figures by multiplying the conversion ratio, 1.49 as follow.

Table 30 The cost of service products per TEU

(Unit: Krw/TEU)

\begin{tabular}{|c|c|c|c|c|c|c|c|}
\hline & & $\begin{array}{c}\text { Local } \\
\text { \&Inter } \\
\text { TMNL } \\
\text { TS } \\
\end{array}$ & $\begin{array}{c}\text { Internal } \\
\mathrm{T} / \mathrm{S}\end{array}$ & $\begin{array}{l}1 \text { Time } \\
\text { Shifting }\end{array}$ & $\begin{array}{l}2 \text { Tim } \\
\text { Shifting }\end{array}$ & $\mathrm{H} / \mathrm{co}$ & $\begin{array}{l}\text { Total } \\
\text { (Avera } \\
\text { ge) }\end{array}$ \\
\hline \multirow{4}{*}{$\begin{array}{l}\text { Total } \\
\text { (Avr.) }\end{array}$} & $\mathrm{Q} / \mathrm{C}$ & 17,921 & 17,921 & 17,921 & 17,921 & 17,921 & 17,921 \\
\hline & RTGC & 24,731 & 12,185 & & 1,408 & & 20,835 \\
\hline & $\mathrm{Y} / \mathrm{T}$ & 15,605 & 15,358 & & 15,112 & & 15,252 \\
\hline & Total & 58,257 & 45,463 & 17,921 & 34,440 & 17,921 & 54,007 \\
\hline \multirow{4}{*}{$\begin{array}{l}\text { Off- } \\
\text { dock }\end{array}$} & $\mathrm{Q} / \mathrm{C}$ & 16,983 & 16,983 & 16,983 & 16,983 & 16,983 & 16,983 \\
\hline & RTGC & 24,339 & 11,533 & & 1,153 & & 19,867 \\
\hline & $\mathrm{Y} / \mathrm{T}$ & 14,979 & 14,682 & & 14,481 & & 14,641 \\
\hline & Total & 56,301 & 43,198 & 16,983 & 32,616 & 16,983 & 51,491 \\
\hline \multirow{4}{*}{$\begin{array}{l}\text { On- } \\
\text { dock }\end{array}$} & $\mathrm{Q} / \mathrm{C}$ & 22,202 & 22,202 & 22,202 & 22,202 & 22,202 & 22,202 \\
\hline & RTGC & 26,664 & 16,811 & & 6,612 & & 25,254 \\
\hline & $\mathrm{Y} / \mathrm{T}$ & 18,499 & 19,202 & & 18,023 & & 18,040 \\
\hline & Total & 67,366 & 58,215 & 22,202 & 46,837 & 22,202 & 65,496 \\
\hline
\end{tabular}

As shown in the Table 30, each average price per TEU of off-dock mode and on-dock mode is analyzed to be Krw 51,491 and 65,496 respectively.

\subsection{Verification}

All the processes of cost analysis have been completed and the final stage to the study is to verify the unit costs.
This study chose two ways of verification as follow.

First, as a common and simple way to verify the unit cost, terminal operators can calculate 'price per TEU' by dividing the total annual cost by the total throughput. This paper allocated total 40,984 Mil/Krw into 745,879 TEU. 40,984 Mil/Krw divided by 745,879 TEU is 54,948 Krw/TEU and there is about $1.7 \%$ of difference between the result of the study, 54,007 Krw/TEU, and the simple calculation, $54,948 \mathrm{Krw}$. The difference mainly comes from the rounding off below the decimal point.

Table 31 Verification by the cost groups

\begin{tabular}{|c|c|c|c|c|c|c|c|}
\hline \multirow{3}{*}{ Cost Item } & \multirow{2}{*}{\multicolumn{3}{|c|}{$\begin{array}{l}\text { Unit Cost for one } \\
\text { move of each } \\
\text { equipment (Krw) }\end{array}$}} & \multicolumn{3}{|c|}{$\begin{array}{l}\text { Total allocated } \\
\text { cost(Mil/Krw) }\end{array}$} & \multirow{3}{*}{ Total } \\
\hline & & & & \multirow{2}{*}{\begin{tabular}{|c|}
$\mathrm{Q} / \mathrm{C}$ \\
508,978 \\
\end{tabular}} & \multirow{2}{*}{$\frac{\text { RTGC }}{1,143,960}$} & \multirow{2}{*}{$\begin{array}{c}\mathrm{Y} / \mathrm{T} \\
513,691 \\
\end{array}$} & \\
\hline & $\mathrm{Q} / \mathrm{C}$ & RTGC & $\mathrm{Y} / \mathrm{T}$ & & & & \\
\hline $\mathrm{Lab}$ & 4,585 & 4,580 & 11,571 & 2,334 & 5,239 & 5,944 & 13,517 \\
\hline Power \& Fuel & 432 & 857 & 712 & 220 & 980 & 366 & 1,566 \\
\hline Mainter & 1,123 & 408 & 765 & 572 & 466 & 393 & 1,431 \\
\hline $\begin{array}{c}\text { Non-operating } \\
\text { cost }\end{array}$ & 340 & 42 & 3,157 & 991 & 3,023 & 622 & 8,635 \\
\hline Rental & 9,814 & 4,106 & 4,861 & 4,995 & 4,697 & 2,497 & 12,189 \\
\hline Overhe & 88 & 9 & 1,451 & 480 & 1,39 & 745 & 3,620 \\
\hline Total & 26,702 & 13,812 & 22,516 & 13,591 & 15,801 & 11,566 & 40,95 \\
\hline
\end{tabular}

On the other hand, if the first way is a top-down approach, the second way is a bottom-up verification. This paper grouped total terminal costs into six categories and each group cost is allocated to the activity of major equipment. Accordingly, it would be possible to sum up all allocated costs and compare the result(40,958 Mil/Krw) with the annual total cost $(40,984 \mathrm{Mil} / \mathrm{Krw})$ as shown in the Table 31 . There was a just $0.07 \%$ of very small difference coming from the rounding off.

In conclusion, it was at least verified that total terminal costs can be allocated into service products based on this ABC approach.

\section{Conclusion}

This paper tried to treat invisible terminal services as a tangible product and calculated production cost based on $\mathrm{ABC}$ approach. At first stage, this paper defined terminal service products as Local, Internal TS, Inter terminal TS, Hatch Cover, 1 time Shifting (Cell to Cell) and 2 times Shifting (cell-Dock-Cell). In the meantime, total terminal costs are grouped into six categories such as Labor cost, Power and Fuel, Maintenance, Rental charge, Non-operating cost and other overhead cost. 
The key point of the study is that this paper regarded the activities of major equipment such as Quay Crane, Rubber Tire Gantry Crane and Yard Tractor as a raw material which is necessary to produce aforementioned products. The result or process of the cost analysis could be different depending on what factors we choose as key activities because all costs will be eventually allocated to the key activities to calculate production cost.

The benefits of the cost analysis using $\mathrm{ABC}$ approach could be summarized as follow.

First, terminal operators could efficiently control their cost through the qualitative approach by eliminating unnecessary cost or reducing less necessary cost.

Second, terminal operators could design different rate systems for feeder lines to support the feeder network which has been moved to container terminals from the closed conventional piers.

Third, this analysis could help terminal operators to pursue productivity improvement and enhance operation efficiency through the cost effective activity.

Last, this approach could help terminal operators develop new services and differentiate the current standardized terminal services to meet customers' various wants and needs even in spite of the cost pressure.

However, there have been few researches that analyze production cost of terminal services based on $\mathrm{ABC}$ approach. So, it was not easy to make a thorough study on the subject and more research has to be done on this topic. Meanwhile, this study requires very detailed internal data and sensitive information to perform realistic study. However, there is a limit to get internal data from terminals, which has been obstacle that keeps this kind of approach from being active and progressed.

Though leaving much to be desired, this study just puts more significance on attempting to link $\mathrm{ABC}$ approach with terminal cost analysis based on actual data for the first time and hopes more research to be done to compensate the defect of the study and to bring progress in this area.

\section{References}

[1] Han, K. H.(2006), "A Study on the Performance Effect of Process Management in Korean Shipping Companies", Paper for Degree of Doctor, Graduate school of Korea Maritime University, pp. 72-84.

[2] Hur, Y. S. and Kim, Y. S.(2009), "Estimated on the Busan Economic Contribution of Container Cargo in Busan Unit(Won/TEU)", Journal of Navigation and Port
Research, Vol. 33, No.3, p.212.

[3] Kwon, Y. J. and Kim, H. W.(2002), "An Analysis of the Cost Structure of Railway Freight Industry in Korea, The Korean Society for Railway.

[4] Lee, J. H.(2009), Yul-Seong Kim, Chang-Hoon Shin(2009), "A Study on Competition Structure among Domestic Container Ports", Journal of Navigation and Port Research, Vol. 33, No.1, p.97.

[5] Rotch, W(1990). "Activity-Based Costing in Service Industries", Journal of Cost Management. Summer ,pp. 4-14.

[6] Stikant Datar, Sunder Kekre, Tridas Mukhopadyay, and Eric Syaan, Overloaded Ocwegwsa(1991), "ActivityBased Cost Analsis of Material Handling in Cell Manufacturing", Journal of Operations Management, Vol, 10, No.1. Jan.

Received 3 August 2011

Revised 16 September 2011

Accepted 21 September 2011 